\title{
Optoelectronic difference-frequency synthesiser: terahertz-waves for high-resolution spectroscopy
}

\author{
L. Aballea and L.F. Constantin ${ }^{\mathrm{a}}$ \\ Laboratoire Phlam, UMR 8523 CNRS, 59655 Villeneuve d'Ascq, France
}

Received: 31 August 2008 / Received in final form: 4 November 2008 / Accepted: 18 November 2008 Published online: 25 December 2008 - (C) EDP Sciences

\begin{abstract}
We demonstrate a simple approach for a difference-frequency synthesiser within the terahertz domain. Two near-infrared CW diode lasers are frequency-locked to the resonances of a high finesse Fabry-Perot cavity using the Pound-Drever-Hall technique. Two acousto-optic modulators driven by RF synthesizers frequency-shift each diode laser and provide continuous tuning, modulation and switching capabilities for the difference-frequency. Terahertz-waves generated by heterodyne mixing in an ultrahighbandwidth photodetector are used to interrogate the rotational transitions in the ground state of the methyl-chloride.
\end{abstract}

PACS. 42.62.Eh Metrological applications; optical frequency synthesizers for precision spectroscopy 33.80.-b Photon interactions with molecules - 85.60.-q Optoelectronic devices - 07.57.-c Infrared, submillimeter wave, microwave and radiowave instruments and equipment

\section{Introduction}

Actually there is an increasing interest in developing coherent sources operating at terahertz frequencies $(0.1-10 \mathrm{THz})$ in order to bridge the electromagnetic gap between electronics and optics [1]. Despite the recent progress with terahertz quantum cascade lasers [2], a compact source that guarantees broad frequency tuning and high spectral quality is a challenging technological issue. The well-established way for broadband $\mathrm{THz}$ generation is the frequency down-conversion of two detuned lasers in a photonic material. Different optical setups have been proposed using non-linear crystals and high-power lasers $[3,4]$, but they are outsized. A promising monolithically integrated $\mathrm{THz}$ semiconductor source is an infrared dual-wavelength quantum cascade laser with giant secondorder nonlinear susceptibility [5]. An alternative way is the difference-frequency generation from two CW nearinfrared lasers heterodyned in a biased photoconductor coupled to an antenna [6]. This device has been recently used in high resolution molecular spectroscopy [7] or imaging [8].

We report some preliminary results on infrared to $\mathrm{THz}$ frequency synthesis using this device. Different molecular rotational lines provide a dense grid of $\mathrm{THz}$ frequency references with an accuracy of a few $\mathrm{kHz}$ and allow the cal-

\footnotetext{
a e-mail: fconstan@lsh.univ-lille1.fr
}

ibration of the difference-frequency over a broad spectral domain. Recently, a frequency standard was proposed at $1.82 \mathrm{THz}$ using a three-photon process in $\mathrm{Ca}^{+}$[9] with an expected precision at $10^{-11}$ level. A fixed-frequency reference signal can be thus obtained by down-conversion from the optical domain [10]. Alternatively, one can use the frequency grid provided by the spectrum of a femtosecond laser that has the repetition rate locked to an optical frequency standard. Phase-locking each laser source used for $\mathrm{THz}$ difference-frequency generation to different lines of an optical frequency comb [11] allows to transfer the metrological features of the optical frequency standard to the $\mathrm{THz}$ domain. Here, the resonances of an optical cavity are used as references for the frequency stabilisation of the lasers with a Pound-Drever-Hall setup [12] and the measurement of molecular lines provides the frequency calibration in the $\mathrm{THz}$ range. The difference-frequency is well-controlled by frequency-shifting the lasers with acousto-optic modulators.

Laser frequency stabilisation on an optical cavity with the Pound-Drever-Hall technique has the advantage to provide a servo loop that is not limited by the response time of the cavity. This is achieved through the phasemodulation of the laser field at a frequency greater than the cavity linewidth. The error signal is recovered by subsequent phase-sensitive demodulation of the heterodyne beat between the field reflected by the input mirror of the cavity and the leakage of the cavity-stored field. The error 


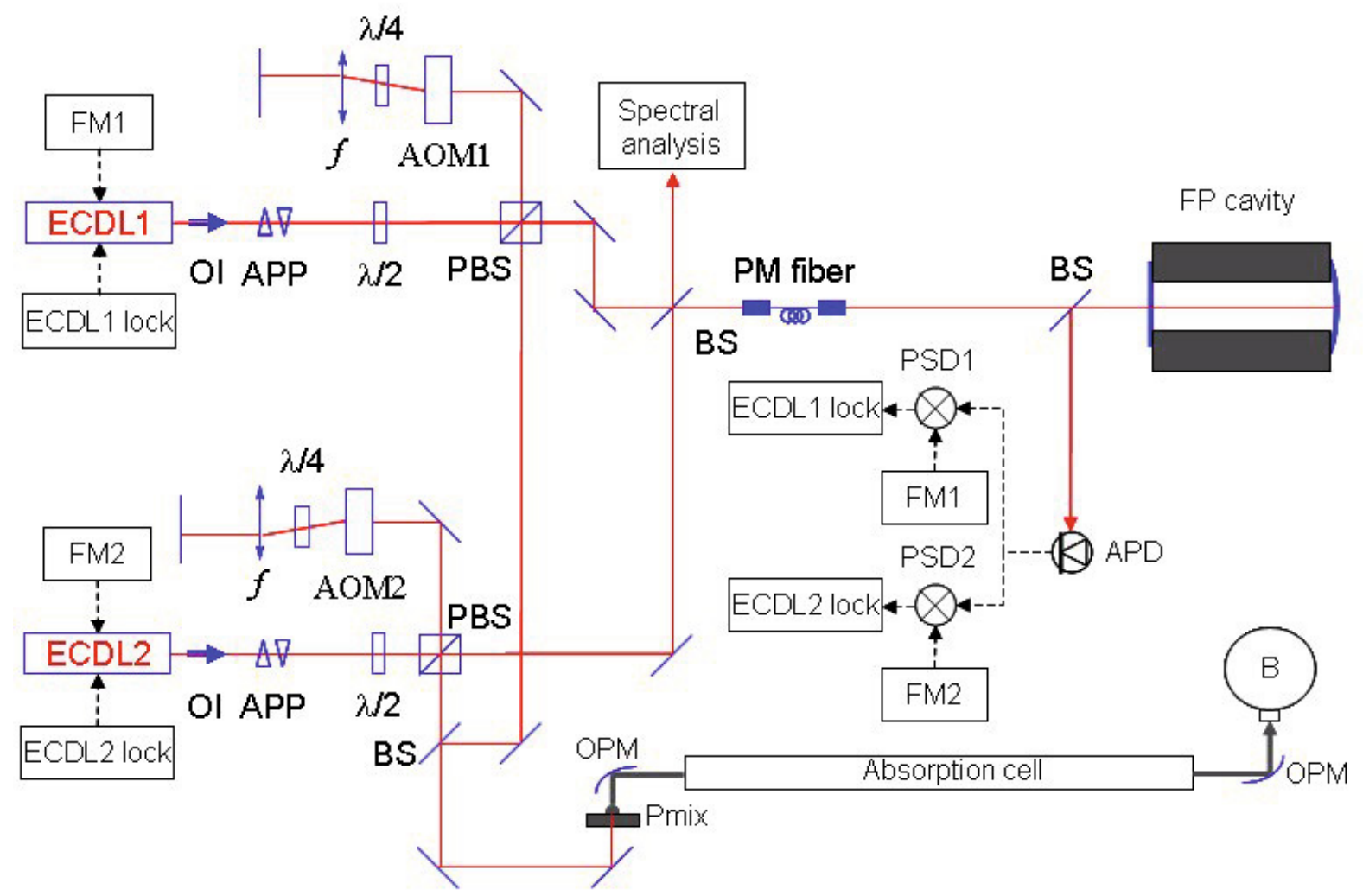

Fig. 1. (Color online) Experimental setup. Solid and dashed lines represent respectively optical and electrical signals. AOM acousto-optical modulator, $\lambda / 4$ and $\lambda / 2$ waveplates, FP Fabry-Perot, P mix photomixer, B bolometer, OPM off-axis parabolic mirror. Refer to the text for details.

signal measures phase fluctuations of the laser at high frequency comparing to the cavity field that serves as phase reference. The performances of a well-designed servo loop will be dictated by the accuracy of the modulation technique and of the laser's phase-correcting transducer.

\section{Experimental setup}

The experimental setup is shown in Figure 1. Two extended-cavity diode lasers (ECDL) in the Littrow configuration are emitting around $820 \mathrm{~nm}$ with an optical power of $80 \mathrm{~mW}$. The free-running ECDL emission linewidth is $1.5 \mathrm{MHz}$ full with at half maximum (FWHM) at an integration time of $1 \mathrm{~s}$. The laser cavity is driven with a PZT transducer that allows tuning the laser radiation without mode-hops over up to $30 \mathrm{GHz}$. Each laser beam is coupled to a two-stage optical isolator (OI) and an anamorphic prism pair (APP) that ensure linear polarisation and similar spatial shape for the laser beams. A half-waveplate and a polarising beamsplitter (PBS) allow redirecting an adjustable amount of laser power from each ECDL to the optical cavity and to the difference-frequency mixer. The diode laser beams are spatially overlapped on a beamsplitter plate (BS). One of the emerging beams allows frequency analysis of the heterodyne beat between the diode lasers on a photodiode with a spectrum analyser. Alternatively, their optical frequency can be measured using a lambdameter. The other emerging beam is injected in an AFC-connectorised polarisation-maintaining (PM) optical fiber. Laser beam exiting the optical fiber is collimated with an aspheric lens and mode-matched with a telescope to the $\mathrm{TEM}_{00}$ mode of the Fabry-Perot cavity. The total incident power from each diode laser is $6 \mu \mathrm{W}$ and $\sim 48 \%$ is coupled to the cavity's mode.

The design of the optical cavity aims to suppress its free spectral range (FSR) jitter and drift through an efficient damping of the acoustic noise and the thermal isolation. The cavity consists in a plane and a concave mirror (radius of curvature $1 \mathrm{~m}$ ) bonded on a $20 \mathrm{~cm}$ long ultralow expansion glass (ULE) cylinder. The cavity lies along the axis of a cylindrical vacuum chamber that is maintained at a pressure less than $10^{-5} \mathrm{~Pa}$ and temperaturestabilised at $320 \mathrm{~K}$. The ULE spacer rests on a $V$-shaped invar plate placed inside two concentric cylindrical aluminium shields. All these elements are isolated each from another and from the vacuum chamber with Viton joints. The optical performances of the cavity are characterised through its finesse and transmission coefficient. The measurement of the exponential decay of the field stored in the cavity yields a cavity linewidth of $234 \mathrm{kHz}$ FWHM 
and a finesse of 3200. Direct power measurements provide a transmission coefficient of $\sim 36 \%$.

Local oscillators at $F M_{1}=20 \mathrm{MHz}$ (respectively at $F M_{2}=24 \mathrm{MHz}$ ) provide a signal that is capacitively coupled to ECDL1 (respectively ECDL2) driving current to generate optical sidebands that are about $10 \%$ of the optical carrier. The light reflected from the cavity is redirected by a beamsplitter to an avalanche photodiode detector (APD). The laser field reflected from the in-coupling mirror of the cavity and the field leaking from the cavity produce beatnote signals at $F M_{1}$ and $F M_{2}$ that are used in the feedback branches for ECDL1 and ECDL2. The beatnote signal is high-pass filtered, amplified and demodulated with a phase-sensitive detector (PSD). An adjustable phase delay circuit for the beatnote signal allows choosing the suitable quadrature component. The demodulated signal is low-pass filtered ( $3 \mathrm{~dB}$ point at $8 \mathrm{MHz}$ ) to suppress the frequency of the local oscillator and its harmonics and serves as error signal for the feedback loop. The error signal is resistively-coupled to the gate of a field-effect transistor (FET) which is mounted in parallel with the diode laser [13]. Consequently, a part of the laser injection current is bypassed through the FET, providing a mechanism for the control of the laser frequency from dc up to a few $\mathrm{MHz}$.

Figure 2a shows the demodulated error signal as the grating of ECDL1 is tuned. The error signal is recorded for visualisation purpose at low bandwidth with an averaging time constant of $0.1 \mathrm{~ms}$ and displays a $\mathrm{S} / \mathrm{N}$ ratio normalised at 8700 to $1 \mathrm{~s}$. The closed servo loop has a bandwidth limited at $700 \mathrm{kHz}$ that is attributed to the response delay of the FET-diode laser system. The servo loop response is dictated by the cavity bandwidth that acts as low pass filter to the laser frequency variations with a corner frequency at $1 / 2 \times 234 \mathrm{kHz}$. We checked that the in-loop error signal noise is well above the fundamental shot noise limit (at $1 \mu \mathrm{W}$ power incident from each laser on the APD). The stabilisation system operates thus in a gain-limited regime and the in-loop error signal variations estimate well the locked laser noise.

Figure $2 \mathrm{~b}$ shows the beatnote between the diode lasers locked on adjacent modes of the cavity. It has a Lorentzian shape with a linewidth of $84 \mathrm{kHz}$ FWHM. The noise from the reference cavity has a negligible contribution to the measured linewidth. This point is important because when the servo loop is operated in a reference-limited regime, the frequency noise of the beatnote is expected to increase with the number of modes between the locked ECLDs. Thus, the measured linewidth may be still valid for the radiation generated by the heterodyne mixing in the $\mathrm{THz}$ regime.

The phase modulation of the diode laser is associated with a strong residual amplitude modulation (RAM). That contributes to a spurious offset and an asymmetry of the error signal which are visible in Figure $2 \mathrm{a}$ and lead to a frequency shift between the locked laser and the resonance
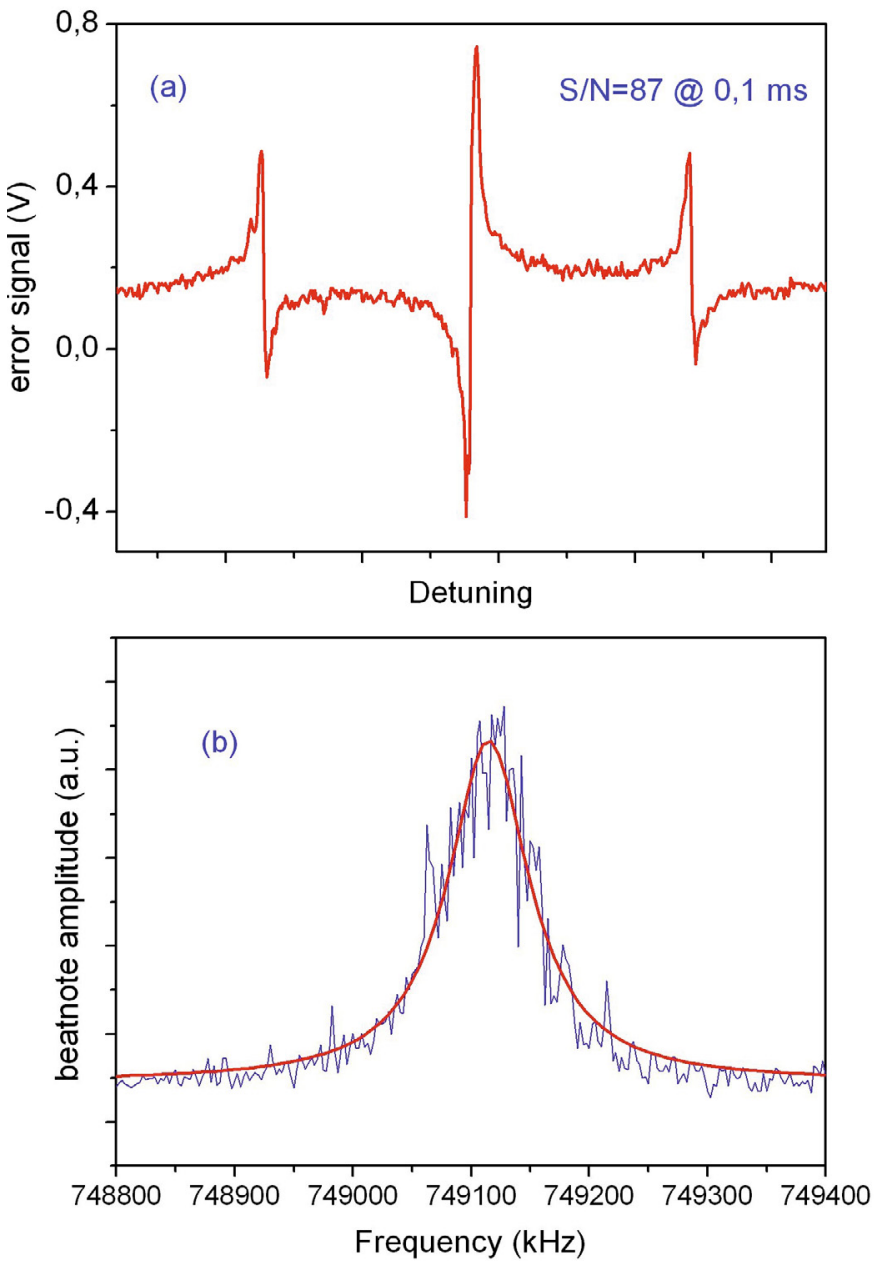

Fig. 2. (Color online) (a) Pound-Drever-Hall error signal. Integration time $0.1 \mathrm{~ms} /$ point, sweep time $52 \mathrm{~ms}$. (b) Beatnote between the diode lasers locked on adjacent cavity modes. RBW $10 \mathrm{kHz}$, recording time $1 \mathrm{~s}$.

of the cavity. We estimate that each of these systematic frequency shifts have a contribution no more than $25 \mathrm{kHz}$.

An important figure of merit of the frequency lock is the reproducibility. 22 measurements of the beatnote between the diode lasers locked on two adjacent modes of the cavity have been carried out during one day. We derive a mean FSR value of $749104 \mathrm{kHz}$ with a standard deviation of $35 \mathrm{kHz}$.

The diode lasers are frequency-shifted with acoustooptic modulators (AOM) driven by radiofrequency synthesizers in a double-pass scheme. The optical setup provides a stable angular deviation of the frequencyshifted light [14] that guarantee the alignment required in difference-frequency generation. Laser beam separated through the polarizing beamsplitter cube is deflected by the AOM in the first-diffraction order to a quarter-wave plate which is associated downstream with a cat's-eye retro-reflector. It relies on a planar mirror located in the focal plane of a $10 \mathrm{~cm}$ focal-length plan-convex lens and 
the laser beam incidence on the AOM is at the focal point of the lens. Light reflected back by the cat's-eye pass through the waveplate, is frequency-shifted again by the AOM, overlaps the incoming laser beam and is transmitted through the polarising beamsplitter cube.

At a given radiofrequency shift, the AOM is oriented such that the incoming laser beam is incident at the Bragg angle. The laser beam profile is Gaussian with a diameter $\sim 1.5 \mathrm{~mm}$ full width $(\mathrm{FW})$ at $1 / e^{2}$ and a divergence $\sim 1.1 \mathrm{mrad} \mathrm{FW}$ at $1 / e^{2}$. The $\mathrm{TeO}_{2}$ modulator is driven at a $\mathrm{RF}$ power of $27 \mathrm{dBm}$. The maximum diffraction efficiency in the double-pass scheme is $35 \%$. A broad change of the radiofrequency shift requires rotating the AOM to keep the Bragg angle incidence, while the cat's eye configuration ensures normal incidence of the laser beam on the planar mirror. The diffraction efficiency drops at 50\% from its maximum value when the radiofrequency is varied in the range $180-360 \mathrm{MHz}$ that defines the overall AOM tuning bandwidth. If the orientation of the AOM is kept fixed, the cat's-eye configuration significantly improves the tuning bandwidth: e.g. around $f_{A O M}=275 \mathrm{MHz}$ the tuning bandwidth is $12 \mathrm{MHz}$ FWHM with a cat's-eye reflector, comparing to $2.3 \mathrm{MHz}$ FWHM with a simple planar mirror retro-reflector. This AOM bandwidth guarantees frequency filtering of the laser sidebands generated for frequency stabilisation.

The setup allows synthesising a heterodyne differencefrequency:

$$
\begin{aligned}
f_{T H z} & =\left(f_{1}+2 f_{A O M 1}+\delta f_{1}\right)-\left(f_{2}-2 f_{A O M 2}+\delta f_{2}\right) \\
& =n F S R+\left(\delta f_{1}-\delta f_{2}\right)+\left(2 f_{A O M 1}+2 f_{A O M 2}\right)
\end{aligned}
$$

by applying to the stabilised diode lasers (tuned at $f_{1}>f_{2}$ ) frequency shifts $f_{A O M 1,2}$ with AOMs working in their + or - first-diffraction order. Slight frequency shifts of the lock point of each diode laser from the corresponding reference are accounted through $\delta f_{1,2}$ terms. The integer $n$ is determined by measuring the differencefrequency with a lambdameter with $50 \mathrm{MHz}$ accuracy. The demonstrated single-AOM tuning bandwidth between $\sim \mathrm{FSR} / 4$ and $\sim \mathrm{FSR} / 2$ allows a twofold tuning bandwidth for $f_{T H z}$. Therefore, one can fine-tune $f_{T H z}$ over one FSR with both AOMs. A computer-based mechanism for relocking a diode laser over consecutive cavity modes would be required for a broader tuning.

$\mathrm{CW} \mathrm{THz}$ radiation is generated by optically heterodyning the AOM-shifted diode laser beams on a photomixer [6]. This device is a photodetector with sub-picosecond response time made from a $2 \mu \mathrm{m}$ GaAs epitaxial layer grown at low temperature on a dielectric substrate. A photoconductive area is defined by a pattern of electrodes that drive a gold spiral antenna. The laser beams are spatially overlapped on a beamsplitter and focused on the electrodes with an aspheric lens. The combined optical power is equally distributed between each laser beam. The photodetector is biased and the ac-photocurrent flowing through the antenna gener-

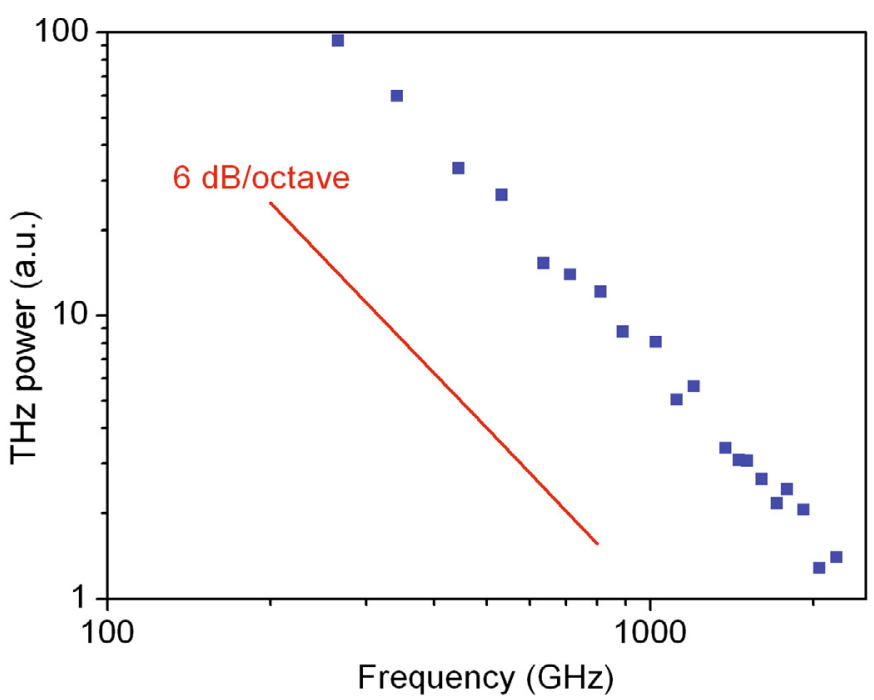

Fig. 3. (Color online) Frequency response of one photomixer biased at $10 \mathrm{~V}$ under a combined optical power of $40 \mathrm{~mW}$; dc-photocurrent $250 \mu \mathrm{A}$.

ates $\mathrm{THz}$ radiation in the substrate. A hyperhemispherical high-resistivity silicon lens mounted in contact with the substrate underneath the antenna extract to freespace the $\mathrm{THz}$ radiation which is directed to a liquidhelium cooled InSb bolometer. Figure 3 shows the typical frequency dependence of the emitted power from one photomixer. The model from [6] predicts that the frequency response of the photomixer has a roll-off with two time constants dictated by the lifetime of the photogenerated carriers and the $R_{A} C$ factor of the antenna. The recorded response displays a roll-off at $-6 \mathrm{~dB} /$ octave up to $2 \mathrm{THz}$ which is attributed to the carriers lifetime. A corner frequency in the response at $1 /\left(2 \pi R_{A} C\right)$ should lie beyond this frequency range.

The $\mathrm{THz}$ beam coming out from the silicon lens is collimated with an off-axis parabolic mirror and coupled to a circular metallic waveguide cell $(1.5 \mathrm{~m}$ long, $3 \mathrm{~cm}$ diameter). The emerging beam from the cell is focused with an off-axis parabolic mirror on the bolometer. Sub$\mu \mathrm{W}$ power is detected for a difference frequency in the $0.1-2 \mathrm{THz}$ range when the photomixer is biased typically at $10 \mathrm{~V}$ and with a combined optical power up to $26 \mathrm{~mW}$.

\section{Comparison with molecular reference lines}

As a demonstration for the difference-frequency synthesis within the $\mathrm{THz}$ domain, we performed high-resolution gas-phase linear absorption spectroscopy on several rotational transitions of the ${ }^{12} \mathrm{CH}_{3}{ }^{35} \mathrm{Cl}$ methyl-chloride isotopomer. For the sake of simplicity, molecular lines are recorded by shifting only ECDL1 frequency with AOM1, while the mixing is performed with radiation from the ECDL2 (AOM2 off). Different modulation techniques are associated for the low-noise detection of the molecular lines. Amplitude modulation (AM) is performed with a 


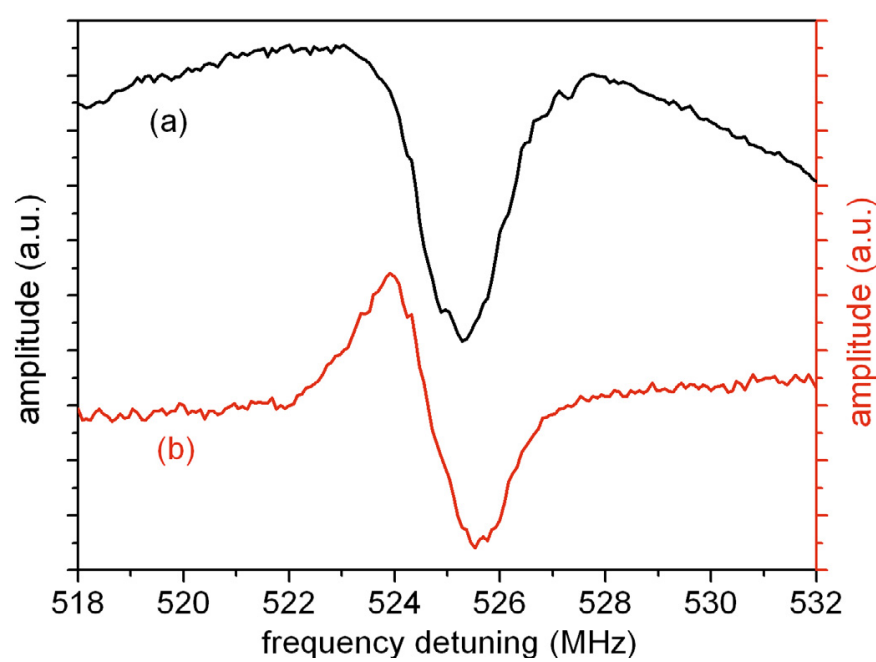

Fig. 4. (Color online) Spectrum of ( $v=0, J: 25 \rightarrow 26, K=5$ ) $\mathrm{CH}_{3} \mathrm{Cl}$ rotation line recorded with (a) amplitude modulation, (b) frequency modulation at $100 \mathrm{kHz}$ and depth of $200 \mathrm{kHz}$. Frequency detuning is $2 f_{A O M 1}$. Pressure $2 \mathrm{~Pa}$, acquisition time $200 \mathrm{~ms} /$ point.

mechanical chopper on the laser beam prior its focusing on the photomixer and the absorption signal is detected with a lock-in amplifier. In this case the absorption line is superposed on a slowly-varying Gaussian-like spectral profile determined by the frequency dependence of the double-pass AOM modulation efficiency. Subtraction of this background signal from the experimental data brings the real molecular absorption lineshape. Alternatively, frequency modulation (FM) of $f_{T H z}$ is performed using the FM capability of the RF synthesizer that drives the AOM and the molecular line is detected at $1-f$ with a lock-in amplifier. This approach has the advantage to shrink the baseline of the absorption spectrum and to diminish the $1 / f$ technical noise.

Figure 4a, 4b show AM- and FM-recorded spectrum of the transition $|v=0, J=25, K=5\rangle \rightarrow \mid v=0$, $\left.J^{\prime}=26, K^{\prime}=5\right\rangle$, where $v$ is the vibrational state and $J, K$ the usual angular momentum quantum numbers. This line is a unresolved quadruplet of four main hyperfine components spanning $0,19 \mathrm{MHz}$ with relative positions $-95.01 \mathrm{kHz},-67.21 \mathrm{kHz}, 64.71 \mathrm{kHz}, 92.52 \mathrm{kHz}$ and relative intensities $0.245,0.255,0.236,0.265$ calculated using the electric-quadrupole coupling constant from [15]. After baseline subtraction, the AM-recorded spectrum was fitted with a Voigt profile.

The main contribution to the recorded linewidth is the Doppler broadening which is $1.2 \mathrm{MHz}$ FWHM. The additional width is due to pressure broadening which is estimated at $0.7 \mathrm{MHz}$ FWHM using the pressure broadening coefficient from [16]. The FM-recorded spectrum exhibits clearly the baseline reduction.
Table 1. Rotational, centrifugal distortion and electric quadrupole coupling constants for the vibrational ground state of ${ }^{12} \mathrm{CH}_{3}{ }^{35} \mathrm{Cl}$, from [15]. $1 \sigma$ uncertainties on the last digits quoted in parentheses.

\begin{tabular}{cc}
\hline Parameter & Value $(\mathrm{MHz})$ \\
\hline$B$ & $13292.87666(6)$ \\
$D_{J}\left(\times 10^{3}\right)$ & $18.09599(39)$ \\
$D_{J K}\left(\times 10^{3}\right)$ & $198.7990(148)$ \\
$H_{J}\left(\times 10^{6}\right)$ & $-0.010148(56)$ \\
$H_{J K}\left(\times 10^{6}\right)$ & $0.3299(49)$ \\
$H_{K J}\left(\times 10^{6}\right)$ & $9.373(147)$ \\
$e Q q$ & $-74.7514(11)$ \\
\hline
\end{tabular}

The frequency of a pure rotational transition is calculated following the formula:

$$
\begin{aligned}
\nu_{T H z}(J, K)= & 2 B(J+1)-4 D_{J}(J+1)^{3} \\
& -2 D_{J K}(J+1) K^{2} \\
& +H_{J}(J+1)^{3}\left((J+2)^{3}-J^{3}\right) \\
& +4 H_{J K}(J+1)^{3} K^{2}+2 H_{K J}(J+1) K^{4}
\end{aligned}
$$

where $B$ is the rotational constant and $D_{J}, D_{J K}, H_{J}$, $H_{J K}, H_{K J}$ are the centrifugal distortion constants listed in Table 1.

We simply estimate the $1 \sigma$ uncertainty for the predicted frequency as the quadratic sum of the $1 \sigma$ error contribution for each rotational constant term. An intensity-averaged frequency shift of the hyperfine structure components is added to the rotational frequency. The least-squares fit of the spectrum with the Voigt profile yields the AOM frequency shift for each rotational line. Table 2 shows the predicted and the observed frequencies for the measured lines. We derive a mean value of the FSR at $749103.2 \mathrm{kHz}$ with a standard deviation of $0.67 \mathrm{kHz}$ (relative uncertainty $9 \times 10^{-7}$ ). Unfortunately, the calibration of the difference-frequency is strongly affected by the systematic frequency shifts of the locked laser. They should be responsible for the significant value observed $(626(14) \mathrm{kHz})$ of the zero-frequency intercept in the linear regression of $\nu_{T H z}+\delta \nu_{H F}-2 f_{A O M 1}$ against $n$. An electronic compensation of the offset of the error signal, as done in [17], in the servo loop of the diode lasers would improve the difference-frequency accuracy.

\section{Conclusion}

Broadband synthesis of $\mathrm{CW} \mathrm{THz}$ radiation is demonstrated from the difference-frequency of two infrared diode lasers locked with the Pound-Drever-Hall technique on an optical cavity. The achieved spectral purity is an order of magnitude better than in previous photomixing experiments [7] and is comparable with that obtained at short timescales for the free-running $\mathrm{THz}$ QCL [2]. The setup 
The European Physical Journal Applied Physics

Table 2. Methyl-chloride lines for the FSR frequency calibration. $1 \sigma$ uncertainties on the last digits quoted in parentheses.

\begin{tabular}{|c|c|c|c|c|c|c|c|c|}
\hline \multicolumn{4}{|c|}{ Quantum numbers } & \multirow{2}{*}{$\begin{array}{l}\text { Calculated frequency } \\
\nu_{T H z}(\mathrm{MHz})\end{array}$} & \multirow{2}{*}{$\begin{array}{l}\text { Hyperfine shift } \\
\delta \nu_{H F}(\mathrm{MHz})\end{array}$} & \multirow[t]{2}{*}{$n$} & \multirow{2}{*}{$\begin{array}{l}\text { Measured frequency }(\bmod \mathrm{FSR}) \\
2 f_{A O M 1}(\mathrm{MHz})\end{array}$} & \multirow{2}{*}{$\begin{array}{l}\text { Calibration } \\
\text { FSR (MHz) }\end{array}$} \\
\hline$J$ & $K$ & $J^{\prime}$ & $K^{\prime}$ & & & & & \\
\hline 9 & 0 & 10 & 0 & $265785.561(3)$ & -0.014 & 354 & 602.743 & 749104.046 \\
\hline 9 & 1 & 10 & 1 & $265781.587(2)$ & -0.013 & 354 & 599.051 & 749103.244 \\
\hline 9 & 2 & 10 & 2 & $265769.666(2)$ & -0.011 & 354 & 587.150 & 749103.184 \\
\hline 9 & 3 & 10 & 3 & $265749.805(2)$ & -0.008 & 354 & 567.002 & 749103.983 \\
\hline 25 & 4 & 26 & 4 & $689792.105(31)$ & -0.001 & 920 & 525.289 & 749102.555 \\
\hline 25 & 5 & 26 & 5 & $689699.456(35)$ & -0.001 & 920 & 617.756 & 749102.357 \\
\hline
\end{tabular}

allows a resolution three orders of magnitude better than that achieved with femtosecond-laser based Fourierlimited terahertz frequency-comb spectroscopy [18]. The measurement of some molecular references pointed out the instrumental errors due to the modulation technique. Improvement of the frequency accuracy and the spectral purity would be achieved through a cautious implementation of the modulation technique and a refined servo loop with high gain at low frequency and wide bandwidth which should operate on a transducer that avoids FM to AM coupling in diode laser. In addition, the proposed AOM-driven difference-frequency generation scheme provides full control of the $\mathrm{THz}$ radiation by using RF synthesizers. A difference-frequency setup with the lasers referenced to a stabilised optical comb would facilitate the access and the control of the radiation from dc to hundreds of $\mathrm{THz}$ for precision molecular spectroscopy.

D. Bacquet contributed significantly for the set up of the Fabry-Perot cavity. We acknowledge K. Blary for providing GaAs photomixers. LFC appreciated fruitful discussions with A. Amy and with Robert James Butcher.
This work was supported by the French Ministry of Research and Education, the Nord-Pas de Calais borough and the CNRS.

\section{References}

1. Phil. Trans. R. Soc. Lond. A 362, 1815 (2004)

2. A. Barkan et al., Opt. Lett. 29, 575 (2004)

3. A.K. Kawase et al., J. Phys. D 35, R1 (2002)

4. T. Tanabe et al., J. Phys. D 36, 953957 (2003)

5. M.A. Belkin et al., Nat. Photon. 1, 288 (2007)

6. E.R. Brown et al., Appl. Phys. Lett. 66, 285 (1995)

7. Pin Chen et al., J. Mol. Spectrosc. 236, 116 (2006)

8. I.S. Gregory et al., Electron. Lett. 40, 143 (2004)

9. C. Champenois et al., Phys. Rev. Lett. 99, 013001 (2007)

10. J.C. Bergquist, H.U. Daniel, Opt. Commun. 48, 327 (1984)

11. Q. Quraishi et al., Opt. Lett. 30, 3231 (2005)

12. R.W.P. Drever et al., Appl. Phys. B 31, 97 (1983)

13. For an example, see Toptica Photonics DL100 Diode Laser System manual, Part 4, Chap. 4.2 (2002), p.18

14. E.A. Donley et al., Rev. Sci. Instrum. 76, 063112 (2005)

15. J. Demaison et al., J. Mol. Spectrosc. 166, 147 (1994)

16. H. Harde et al., J. Opt. Soc. Am. B 11, 1018 (1994)

17. V. Bernard et al., Metrologia 34, 313 (1997)

18. T. Yasui et al., Appl. Phys. Lett. 88, 241104 (2006)

To access this journal online: www.edpsciences.org 